\title{
Nonlinear Dynamical Analysis for an Ethanol Steam Reformer: A Singular Distributed Parameter System
}

\author{
Pedro Reyero-Santiago, Carlos Ocampo-Martinez, Senior Member, IEEE, and Richard D. Braatz, IEEE Fellow
}

\begin{abstract}
This article provides a theoretical analysis of the nonlinear dynamics of an ethanol steam reformer, which has the potential to become a key technology for the creation of a hydrogen economy. A set of nonlinear partial differential equations is analyzed that arise from material and energy balances for an ethanol steam reformer. Although all of the governing equations contain derivatives with respect to both space and time, the nonlinear distributed parameter system is shown to be singular by structural rank analysis. A numerical method is proposed for simulation of the singular dynamical system. The character of the singularity is analyzed for both the distributed parameter systems and the lumped parameter system used in its simulation. The nonlinear spatiotemporal and input-output behavior of the system are analyzed, including by calculation of a nonlinearity measure applicable to singular distributed parameter systems. Although some states are highly nonlinear functions of the control inputs, a linear low-order input-output model with uncertainty description is shown to be suitable for controller design.
\end{abstract}

\section{INTRODUCTION}

The world has seen major interest and growth in the use of energy technology from renewable resources. Some of the technologies of interest have been centered around hydrogen, which is one of the most abundant elements in the universe. Hydrogen technologies have potential for reducing pollutant and greenhouse emissions. Renewable technologies such as wind and photovoltaics can be used to generate hydrogen as a storage medium, which can be used later to generate energy without the production of carbon dioxide or any harmful omissions. The European Union has set targets for reducing harmful emissions (complying with the Paris protocol) and aims to reach at 2030 about $40 \%$ less greenhouse gas emissions (in relation to 1990 levels), while achieving a quota of at least $27 \%$ of renewable energies in the production of electrical energy [1].

Although batteries are increasingly used in automotive transportation, liquid or gaseous fuels have a much higher energy density, which means that vehicles that use fuels spend much less energy transporting the weight of the energy storage medium than vehicles that use batteries. One of the reasons that hydrogen is of interest as a fuel is that it has one of the highest energy densities per unit mass. Hydrogen has

P. Reyero-Santiago and C. Ocampo-Martinez are with the Automatic Control Department, Universitat Politècnica de Catalunya, Institut de Robòtica i Informàtica Industrial (CSIC-UPC), Llorens i Artigas 4-6, 08028 Barcelona, Spain \{pedro.reyero, carlos.ocampo\} @upc.edu

Richard D. Braatz is with the Massachusetts Institute of Technology, E19-551, 77 Massachusetts Avenue, Cambridge, MA 02139, USA braatz@mit.edu

The project leading to these results has received funding from "La Caixa" Foundation through the MIT-Spain Seed Fund ID 100010434, under agreement LCF/PR/MIT17/11820011. an energy-to-weight ratio that is about ten times greater than lithium-ion batteries, offering a greater range while having much lower mass.

Although an infrastructure based on hydrogen would be clean, the option has some drawbacks, mainly related to hydrogen storage and transport [2],[3]. Safe options are available to locally generate enough hydrogen to feed the required energy needs when needed, while avoiding risks associated with hydrogen storage and transport. Reforming is one of the technologies for generating hydrogen when needed, by producing hydrogen from hydrocarbons (ethanol in particular). Different reforming techniques has been proposed, including ethanol steam reforming, oxidation steam reforming, partial oxidation, and decomposition [4]. Given the abundance of existing technologies and infrastructure for the storage and transport of ethanol, ethanol steam reforming is one of the most promising of these techniques.

The use of ethanol steam reformers (ESRs) for the hydrogen production requires the design of efficient and reliable control strategies for ensuring the efficient operation of the device. This latter aspect is a key factor for the integration of such devices into complex and smart grids for clean energy production. Relatively few studies consider the optimal design of control strategies for ethanol steam reformers based on mathematical models. Some studies only consider the steady-state behaviour of the ESR to design proportional-integral-derivative (PID)-based decoupled control loops while ignoring physical and operational constraints (e.g., [5],[6]). Other studies consider the design of predictive controllers for hydrogen outflow regulation while taking into account constraints in inputs, states, and outputs (e.g., [7],[8],[9]). Some of the studies rely on the use of linear process models, which can show poor accuracy in general. Other studies use nonlinear process models, which have higher computational cost when computing the control law, due to the complexity of nonlinear models for ethanol steam reformers.

Past studies have proposed state feedback-based control strategies, which require the design of state estimators. An alternative approach [10] that did not require a state estimator and had low on-line computational cost was a fast model predictive controller based on a linear input-output approximation of the ESR nonlinear model. The online MPC calculations were computable in real time.

Here we report a critical assessment of the extent of nonlinearity for ESR based on its full nonlinear model, which is represented by a system of nonlinear partial differential equations (PDEs) arising from material and energy balances. 


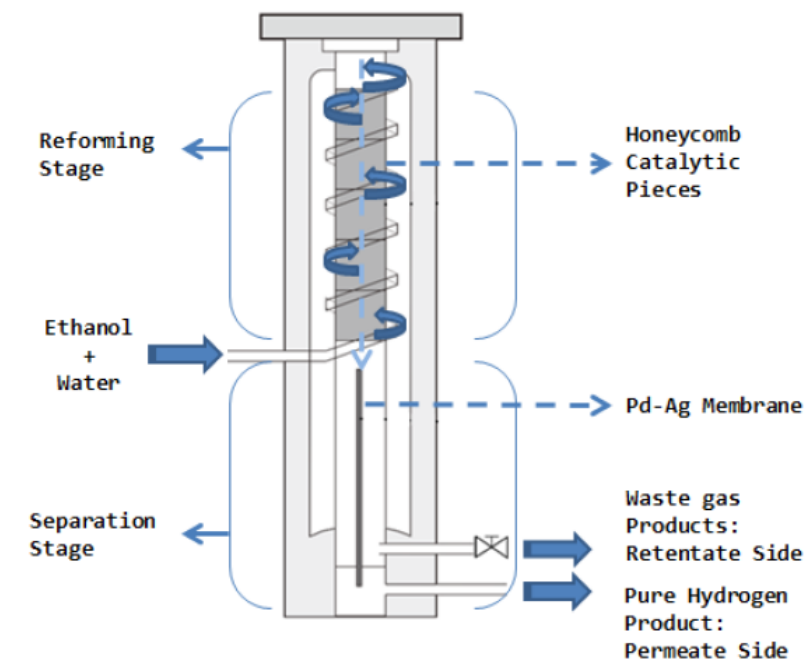

Fig. 1. Staged membrane reactor scheme

The main contribution is a detailed analysis of whether linear or nonlinear process models are most appropriate for the model-based control of ethanol steam reformers. Several approaches are taken to analyzing the nonlinear dynamics based on the full set of PDEs derived by first principles (aka physics). We discover that the physics-based distributed parameter model for the ethanol steam reformer is actually a singular system [11], which motivates the definition of a nonlinearity measure that is applicable to singular distributed parameter systems while having manageable computational cost.

The remainder of this article is organized as follows. Section II describes the ethanol steam reformer system under consideration including the involved chemical reactions and the resultant nonlinear model. Section III analyzes the structural rank of the mass matrix of the system of governing equations, which shows that the system is singular, and describes the numerical method used to solve the systems of differential equations. Section IV defines a nonlinearity measure applicable to singular distributed parameter systems, and applies the measure to the model for the ethanol steam reformer. Section V brings the results together to discuss whether linear or nonlinear models are most appropriate for the control of ethanol steam reformers. Finally, Section VI summarizes the main conclusions.

\section{SYSTEM DESCRIPTION}

This article considers an ethanol steam reformer (ESR) as a nonlinear dynamical system that integrates two process unit operations in series: $(i)$ a reformer stage consisting of a catalytic ethanol steam reactor, and (ii) a separation stage that incorporates a selective membrane through which hydrogen can permeate [8]. Ethanol is reformed with water in the reactor, to produce a mixture of gases from which hydrogen is then separated. The whole process takes place inside of a single integrated module, called a staged membrane reactor [12].
The chemical reactions that occur in the ethanol steam reformer are [9]

$$
\begin{aligned}
\mathrm{C}_{2} \mathrm{H}_{5} \mathrm{OH} & \longrightarrow \mathrm{CH}_{3} \mathrm{CHO}+\mathrm{H}_{2} \\
\mathrm{C}_{2} \mathrm{H}_{5} \mathrm{OH} & \longrightarrow \mathrm{CO}+\mathrm{CH}_{4}+\mathrm{H}_{2} \\
\mathrm{CO}+\mathrm{H}_{2} \mathrm{O} & \longrightarrow \mathrm{CO}_{2}+\mathrm{H}_{2} \\
\mathrm{CH}_{3} \mathrm{CHO}+3 \mathrm{H}_{2} \mathrm{O} & \longrightarrow 2 \mathrm{CO}_{2}+5 \mathrm{H}_{2}
\end{aligned}
$$

The chemical reactions in (1) occur in a tubular packed-bed reactor with a single inlet and a single outlet.

Ethanol dehydrogenates into acetaldehyde and hydrogen (1a), and acetaldehyde is further reformed with water into carbon dioxide and hydrogen (1d). In parallel, an undesired reaction occurs in which ethanol is decomposed into carbon monoxide, methane, and hydrogen (1b). The cobalt catalyst also causes the water-gas shift equation (1c) to occur during the usual operating conditions. Waste gases are kept in the retentate side by a paladium-silver ( $\mathrm{Pd}-\mathrm{Ag}$ ) membrane, through which hydrogen permeates.

Some reasonable and somewhat overlapping assumptions for the mathematical modeling of the ethanol steam reformer are [9]

- The concentrations in the ethanol steam reformer are well described as being two plug-flow units in series (a tubular reactor followed by a tubular membrane separator). The assumption is reasonable for the packed bed reactor, since the solid catalyst particles redistribute the fluid flow in the radial direction. This assumption is reasonable for the membrane separator due to its relatively small diameter.

- The radial dependence of the pressure and temperature is negligible in the tubular reactor and membrane separator. The former assumption is reasonable because there is no or minimal flow or friction in the radial direction, and the latter assumption is reasonable due to the short length scale in the radial direction relatively to the axial direction.

- The fluid is completely radially mixed at each axial position in the tubular reactor and membrane separator. This assumption is not needed to derive the mathematical model, due to the above assumptions, but is a simplified conceptual model often used by chemical engineers to quickly derive the model equations. These assumptions allow the dependence of the velocity in the radial direction to be neglected-instead, the velocity is taken to be average velocity in the axial direction.

- The ideal gas assumption is valid in the operating pressure range, that is, $P(V / n)=R T$ where $P$ is the gas pressure, $V / n$ is the volume per unit moles of gas, $R$ is the ideal gas constant, and $T$ is temperature. This assumption is valid for the relatively low pressures that occur in ethanol steam reformers. Removing this assumption would only require inserting a nonideality constant $Z$ in various places in the model equations ( $Z=1$ for an ideal gas).

- The effects of molecular diffusion in the axial direction are negligible with respect to convection in the axial 
direction. The reasonableness of this assumption can be confirmed by calculation of the Péclet number, which is equal to the length of the vessel multiplied by the mean flow velocity divided by the molecular diffusivity. This assumption holds when the Péclet number is large, which occurs in most industrial applications. This assumption allows the dropping of terms containing second-order spatial derivatives with respect to the axial direction in the nonlinear partial differential equations that model the process.

These assumptions result in the mathematical model for the two process units being a function of the time and the axial direction, that is, to have only one spatial dimension in the physics-based distributed parameter model for the nonlinear dynamics of the system.

For the reforming stage, the molar conservation equations for the seven $j \in\{1, \ldots, 7\}$ species $\left(\mathrm{C}_{2} \mathrm{H}_{5} \mathrm{OH}, \mathrm{H}_{2} \mathrm{O}, \mathrm{CH}_{4}, \mathrm{H}_{2}\right.$, $\mathrm{CO}, \mathrm{CO}_{2}, \mathrm{CH}_{3} \mathrm{CHO}$ ) are described by a set of seven nonlinear differential equations, initial conditions, and boundary conditions:

$$
\begin{aligned}
p \frac{\partial F_{j}}{\partial t}-p & \frac{F_{j}}{\sum_{k} F_{k}} \sum_{k} \frac{\partial F_{k}}{\partial t}-\frac{p}{T} F_{j} \frac{\partial T}{\partial t} \\
& =R T \sum_{k} F_{k}\left(\sum_{i} \nu_{j, i} r_{i}\left(\left\{F_{k}\right\}\right)-\frac{1}{A} \frac{\partial F_{j}}{\partial z}\right), \\
F_{j}(z, 0) & =F_{j, 0}(z), \quad \forall z \in\left[0, \ell_{1}\right], \\
F_{j}(0, t) & =F_{j, \text { in }}(t), \quad \forall t \geq 0,
\end{aligned}
$$

where $i \in\{a, b, c, d\}$ is the respective reaction index according to (1), $k \in\{1, \ldots, 7\}$ refers to summations over all species, $t$ and $z$ are the time and axial position, respectively, $F_{j}$ is the molar flow (in $\mathrm{mol} / \mathrm{min}$ ) of the $j$ th species, $F_{j, 0}$ is the molar flow of the $j$ th species at time $t=0, F_{j \text {,in }}$ is the molar flow of the $j$ th species at the reactor inlet ( $z=0$ ), $\ell_{1}$ is the axial length (in $\mathrm{m}$ ) of the reactor, $\nu_{j, i}$ is the stoichiometric coefficient (dimensionless) of the $j$ th species in the $i$ th reaction, $r_{i}$ is the rate of the $i$ th chemical reaction, $p$ is the gas pressure, and $A$ is the cross-sectional area of the reactor.

Here, the model equations were reformulated in terms of molar flow rates, which reduces the number of states by about half and results in a mathematical structure that is more amenable to theoretical analysis of the process dynamics. The expressions for the chemical reaction rates are described by elementary kinetics [8],[9]:

$$
\begin{aligned}
r_{a} & =k_{a} P_{\mathrm{C}_{2} \mathrm{H}_{5} \mathrm{OH}}, \\
r_{b} & =k_{b} P_{\mathrm{C}_{2} \mathrm{H}_{5} \mathrm{OH}}, \\
r_{c} & =k_{c}\left(P_{\mathrm{CO}} P_{\mathrm{H}_{2} \mathrm{O}}-\frac{P_{\mathrm{CO}_{2}} P_{\mathrm{H}_{2}}}{k_{W G S}}\right), \\
r_{d} & =k_{d} P_{\mathrm{CH}_{3} \mathrm{CHO}} P_{\mathrm{H}_{2} \mathrm{O}}^{3}, \\
k_{W G S} & =e^{\frac{4577.8}{T}-4.33}, \\
k_{i} & =k_{\infty i} e^{-E_{a_{i}}\left(\frac{1}{R T}-\frac{1}{R T_{\mathrm{ref}}}\right)},
\end{aligned}
$$

where $P_{j}$ is the partial pressure (in bar) of the $j$ th component, $T$ is the temperature (in $\mathrm{K}$ ), $T_{\text {ref }}$ is the reference temperature, equal to $773 \mathrm{~K}$, and $R$ is the ideal gas constant (in $\mathrm{Pa} \mathrm{m}^{3} /(\mathrm{mol} \mathrm{K})$ ).

The membrane separator contains no catalyst and negligible chemical reactions occur in that process unit. The separator is modeled by seven molar conservation equations as in (2a), but with the reaction rates term replaced by zero for all species except for hydrogen, for which the term is is replaced by the flow rate of hydrogen per volume unit that permeates/leaves through the membrane, i.e.,

$$
\begin{aligned}
p \frac{\partial F_{\mathrm{H}_{2}}}{\partial t}-p & \frac{F_{\mathrm{H}_{2}}}{\sum_{k} F_{k}} \sum_{k} \frac{\partial F_{k}}{\partial t}-\frac{p}{T} F_{\mathrm{H}_{2}} \frac{\partial T}{\partial t} \\
& =R T \sum_{k} F_{k}\left(-\frac{F_{\mathrm{H}_{2}, p e r m}}{\Delta V}-\frac{1}{A} \frac{\partial F_{\mathrm{H}_{2}}}{\partial z}\right) .
\end{aligned}
$$

In the membrane separator, the boundary conditions come from the continuity of the flow rates between the stages (reforming outlet minus separation inlet). Sievert's law [12] is used to model the mass transfer mechanism for hydrogen permeating through the membrane per unit area, i.e.,

$$
\begin{aligned}
\frac{F_{\mathrm{H}_{2}, \text { perm }}}{\pi D_{m} \Delta z} & =\frac{P_{e}}{\delta}\left(\sqrt{P_{H_{2, r}}}-\sqrt{P_{H_{2, p}}}\right), \\
P_{e} & =P_{e, 0} e^{-\frac{E_{a}}{R T}},
\end{aligned}
$$

where $P_{e}$ and $P_{e, 0}$ are the permeability of the membrane and the permeability pre-exponential factor, respectively (both in

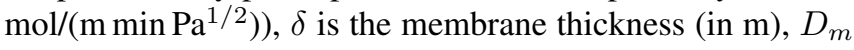
is the membrane diameter (in $\mathrm{m}$ ), $F_{\mathrm{H}_{2}, \text { perm }}$ is the molar flow rate of hydrogen permeating the membrane (in $\mathrm{mol} / \mathrm{min}$ ), $P_{H_{2, r}}$ and $P_{H_{2, p}}$ are the $\mathrm{H}_{2}$ partial pressure (in $\mathrm{Pa}$ ) at the retentate and permeate sides, respectively, and $E_{a}$ is the apparent activation energy (in $\mathrm{J} / \mathrm{mol}$ ). All of these parameters have been fitted to experimental data from an ethanol steam reformer using the procedure described in [9].

At any given point, the concentration of the $j$ th species, $C_{j}$, and the flow's velocity $v$ can be computed from

$$
\begin{aligned}
C_{j} & =\frac{F_{j}}{\sum_{k} F_{k}} \frac{p}{R T}, \\
v & =\frac{1}{A} \sum_{k} F_{k} \frac{R T}{p} .
\end{aligned}
$$

The spatiotemporal dynamics of the ESR temperature are described by the energy conservation equation [8]

$$
\begin{array}{r}
\frac{p}{R T} \frac{\sum_{j} C_{v_{j}} F_{j}}{\sum_{j} F_{j}} \frac{\partial T}{\partial t}=U \beta\left(T_{f}-T\right)-\frac{R T}{A} \sum_{j} \frac{\partial F_{j}}{\partial z} \\
-\frac{1}{A}\left(\sum_{j} C_{p_{j}} F_{j}\right) \frac{\partial T}{\partial z}-\sum_{j} \sum_{i} r_{i}\left(\left\{F_{j}\right\}\right) \Lambda,
\end{array}
$$

with $\Lambda=\Delta H_{i}-\nu_{j, i} R T$ and initial and boundary conditions

$$
\begin{aligned}
& T(z, 0)=T_{0}(z), \quad z \in\left[0, \ell_{1}\right], \\
& T(0, t)=T_{\text {in }}(t), \quad \forall t \geq 0,
\end{aligned}
$$

where $T_{0}$ is the temperature at the initial time $t=0, T_{\text {in }}$ is the temperature of the inlet gases, $T_{f}$ is the temperature (in K) of the furnace that surrounds the tubular units, $U$ is the overall 
heat transfer coefficient (in $\mathrm{J} /\left(\mathrm{m}^{2} \mathrm{~s} \mathrm{~K}\right)$ ), $\beta$ is the area per reactor volume where heat is being transferred (in $\mathrm{m}^{2} / \mathrm{m}^{3}$ ), which is equivalent to $4 / \gamma$, with $\gamma$ being the catalytic reactor diameter (in m), $c_{v_{j}}$ and $c_{p_{j}}$ are heat capacities of the $j$ th species (in $\mathrm{J} /(\mathrm{mol} \mathrm{K}))$, and $\Delta H_{i}$ is the heat of the $i$ th reaction (in $\mathrm{J} / \mathrm{mol}$ ).

The derivation of the energy conservation equation in (8) makes assumptions that correspond to species conservation equations, e.g., (i) the effects of thermal diffusion in the axial direction is negligible relative to convection in the axial direction, and (ii) the dependence of the temperature on the radial direction is negligible.

For the separator, temperature is modeled as in (8), but taking into account the change in the material balance for the second stage, i.e.,

$$
\begin{array}{r}
\frac{p}{R T} \frac{\sum_{j} C_{v_{j}} F_{j}}{\sum_{j} F_{j}} \frac{\partial T}{\partial t}=U \beta\left(T_{f}-T\right)-\frac{1}{A} R T \sum_{j} \frac{\partial F_{j}}{\partial z} \\
-\frac{1}{A}\left(\sum_{j} C_{p_{j}} F_{j}\right) \frac{\partial T}{\partial z}+\frac{F_{\mathrm{H}_{2}, p e r m}}{\Delta V} U_{\mathrm{H}_{2}},
\end{array}
$$

where $U_{\mathrm{H}_{2}}$ is the internal energy of $\mathrm{H}_{2}$.

\section{PDAE ANALYSis AND NumericAl METhoD}

\section{A. Structural analysis}

The set of eight equations defined by the seven species' material balances (2a) and the energy balance (8) can be written in vector form as

$$
M(\vec{x}, t) \frac{\partial \vec{x}}{\partial t}=f\left(\vec{x}, \frac{\partial \vec{x}}{\partial z}, t\right),
$$

where $M(\vec{x}, t)$ is the mass matrix for the distributed parameter system, and $\vec{x}$ is the state vector that contains the molar flows $F_{j}$ and the temperature $T$.

All of the differential equations defining the nonlinear dynamical system contain derivatives with respect to time $t$ and axial position $z$, which would suggest that the model is a system of PDEs. Here, we show that the nonlinear model actually defines a singular/descriptor system, as the mass matrix $M(\vec{x}, t)$ is singular for all $t$ and $z$. In other words, the mass matrix has a structural rank of seven, that is, rank deficient by one.

From (2a) and (8), the mass matrix can be shown to have the structure in (12), where the terms $A_{i}, B_{i}$, and $K$ are

$$
\begin{aligned}
A_{i} & =\frac{p F_{i}}{\sum_{k} F_{k}}, \\
B_{i} & =\frac{p F_{i}}{T}, \\
K & =\frac{p}{R T} \frac{\sum_{k} C_{v_{k}} F_{k}}{\sum_{k} F_{k}} .
\end{aligned}
$$

Inspection of the last row of the matrix $M$, which corresponds to the energy balance, and the facts that $K \neq 0$ and all of the other elements in the last row are zero, imply that the inclusion of this last equation in the model does not affect whether the mass matrix is rank deficient. Thus, the potential rank deficiency can be assessed by analysis of the $7 \times 7$ sub-matrix corresponding to the molar flow states without considering the temperature. The addition of all coefficients in any column of the $7 \times 7$ submatrix gives

$$
\sum_{i} M_{7 \times 7}(i, j)=p-\sum_{i} A_{i}=p-\sum_{i} p \frac{F_{i}}{\sum_{k} F_{k}}=0
$$

i.e., the vector of ones is in the left null-space of the $M_{7 \times 7}$ matrix. In other words, adding all seven sub-equations yields a row of zeros in the sub-matrix.

For the original $8 \times 8$ mass matrix, the linear combination of equations from the system model that yield a row of zeros in the $M$ matrix is

$$
1 \times(\text { Eq. } 2 \mathrm{a})+\cdots+1 \times(\text { Eq. } 2 \mathrm{~g})+\frac{\sum_{i} B_{i}}{K} \times(\text { Eq. 11) }
$$

which proves the rank deficiency of the mass matrix for the nonlinear dynamical system (11).

This analysis shows that the nonlinear dynamical system is a singular system. The system of governing equations are well-posed, but the singularity in the mass matrix indicates that its dynamics will behave like a PDAE system rather than as a PDE system. This observation has several important implications both in the theoretical analysis of its dynamics and in the numerical simulation of the system.

With further theoretical analysis, the mass matrix can be shown to be rank deficient by only one dimension, which implies one source for the singularity. Such singularities in a chemical process are typically associated with a very fast dynamic time constant that has been approximated as being instantaneous to simplify the model equations. For this process, (7) replaces a very fast time scale $(<0.01 \mathrm{~s})$ with a time scale of 0 . While this approximation is very accurate for this system and would not significantly affect the states or outputs, and is desirable for significantly simplifying the model equations, the approximation turns what would be a regular system into a singular system.

\section{B. Finite-difference spatial discretization}

In order to numerically simulate the process model, the spatial derivatives of the states are approximated using firstorder finite backward differences

$$
\frac{\partial x}{\partial z} \approx \frac{x(k)-x(k-1)}{\Delta z}
$$

where 200 discretization points have been used for each stage of the reactor, which was set as being just large enough to have high enough accuracy in the simulated variables to significantly affect the results. With that choice, the PDAE system (11) becomes a system of $8 \times 200=1600$ ordinary differential-algebraic equations in time. A first-order spatial discretization was used because that choice is easy to implement and is highly numerically stable for this particular class of hyperbolic equations. 


$$
M=\left[\begin{array}{cccccccc}
p-A_{1} & -A_{1} & -A_{1} & -A_{1} & -A_{1} & -A_{1} & -A_{1} & -B_{1} \\
-A_{2} & p-A_{2} & -A_{2} & -A_{2} & -A_{2} & -A_{2} & -A_{2} & -B_{2} \\
-A_{3} & -A_{3} & p-A_{3} & -A_{3} & -A_{3} & -A_{3} & -A_{3} & -B_{3} \\
-A_{4} & -A_{4} & -A_{4} & p-A_{4} & -A_{4} & -A_{4} & -A_{4} & -B_{4} \\
-A_{5} & -A_{5} & -A_{5} & -A_{5} & p-A_{5} & -A_{5} & -A_{5} & -B_{5} \\
-A_{6} & -A_{6} & -A_{6} & -A_{6} & -A_{6} & p-A_{6} & -A_{6} & -B_{6} \\
-A_{7} & -A_{7} & -A_{7} & -A_{7} & -A_{7} & -A_{7} & p-A_{7} & -B_{7} \\
0 & 0 & 0 & 0 & 0 & 0 & 0 & K
\end{array}\right]
$$

\section{DAE simulation}

The singularity of the mass matrix for the original 8 model equations results in singularity of 200 dimensions in the $1600 \times 1600$ mass matrix of the resulting 1600 equations in time, implying that the system of 1600 equations is a singular system with high dimensionality in the singularity. Although each equation contains a derivative with respect to time, the singularity results in this being a system of ordinary differential-algebraic (DAE) equations. This DAE system, which has the mass matrix $M$ being a function of the states, was solved using MATLAB's ode15s solver. A differential index analysis applied to the DAE implied that the differential index is one, which is solvable using that solver.

The singular character of the DAE system requires the computation of physically consistent initial conditions before the results of simulating the dynamics of the DAE system to be valid. Physically consistent initial conditions for the full nonlinear dynamical system can be obtained through several methods:

1) Setting all time derivatives in (2a) and (8) to zero and numerically solving the resulting ODEs in which the independent variable is the spatial dimension.

2) Numerically solving the nonlinear dynamical system of equations $f\left(\vec{x}, \frac{d \vec{x}}{d z}, t\right)=0$ that arises from setting the time derivative of the state vector in (11) to zero.

3) By dynamically simulating the larger-dimension DAE system for a constant input until steady state is achieved.

Method 1 is easy to implement and can compute initial conditions for the original distributed parameter model without incurring any inaccuracies in the approximation caused by use of first spatial discretization. However, the fact that initial conditions must be consistent with the DAE system means that a different set of initial conditions are required, which take into account this explicit discretization in space used to derive the DAEs. We bypassed this situation by defining a new mass matrix $M^{\prime}=M+\epsilon I$, where $I$ is the $1600 \times 1600$ identity matrix. The system simulated with $M^{\prime}$ as the mass matrix is nonsingular, which coverts the DAE system to an ODE system for which any value of the state vector is a consistent initialization for the ODE system. We initialized the ODE system from the solution of the spatial ODEs in Method 1). After simulating for enough time for a constant input, the system's state approaches steady state, and the obtained states are also a steady state for the DAE system.
The ODE and DAE systems with 1600 states have the same steady state because the addition of terms involving time derivatives does not change the steady state, where those derivatives are zero. The mass matrix $M^{\prime}$ was then switched back to the original mass matrix $M$ to simulate the dynamics of the singular system.

\section{Step responses}

For the control of the ethanol steam reformer, the control inputs are the inlet flows of ethanol and water, while the output is the permeated volumetric flow of hydrogen,

$$
Q_{\mathrm{H}_{2}, p e r m}=\frac{R T_{a t m}}{p_{a t m}} F_{\mathrm{H}_{2}, p e r m},
$$

where $T_{a t m}$ and $p_{a t m}$ are the atmospheric temperature and pressure, respectively. The total permeated molar flow of hydrogen was computed as

$$
F_{\mathrm{H}_{2}, \text { perm }}=\int_{0}^{l_{2}} \frac{F_{\mathrm{H}_{2}, p e r m}}{\Delta z} d z,
$$

where the integral was computed using Simpson's rule. Our interest is control-relevant models over the entire range of the control inputs, so a staircase function was used to analyze the extent of nonlinearity in the input-output behavior of the distributed parameter system.

\section{NONLINEARITY MEASURE}

In order to quantify nonlinearity of dynamical systems, several different measures have been proposed across the related literature [13]. In this case, two nonlinearity measures were used to assess input-output nonlinearity. Both measures are based on an optimization over sampled sets of models obtained with the normalized staircase response of the system inside of the range of operation defined by the physical constraints

$$
\begin{aligned}
& 1.8 \times 10^{-3} \leq u_{e t h} \leq 2.4 \times 10^{-3} \quad[\mathrm{~mol} / \mathrm{min}], \\
& 8.9 \times 10^{-3} \leq u_{\text {wat }} \leq 10.8 \times 10^{-3} \quad[\mathrm{~mol} / \mathrm{min}]
\end{aligned}
$$

Both nonlinearity measures are based on the definition

$$
\Phi_{N}=\inf _{L \in \mathcal{L}} \sup _{\left(\mathbf{u}, \mathbf{x}_{N, 0}\right) \in \mathcal{S}} \frac{\left\|L\left[\mathbf{u}, \mathbf{x}_{L, 0}\right]-N\left[\mathbf{u}, \mathbf{x}_{N, 0}\right]\right\|}{\left\|N\left[\mathbf{u}, \mathbf{x}_{N, 0}\right]\right\|},
$$

where $\mathbf{u}$ and $\mathbf{x}$ are the system inputs and states; $\|\cdot\|$ is a suitable norm in the space of system outputs $\mathcal{Y} ; N$ : $\mathcal{U}_{a} \times \mathcal{X}_{a, 0} \rightarrow \mathcal{Y}$ is the operator for a nonlinear dynamical system with output signals $\mathbf{y}_{N} \in \mathcal{Y}_{a} \subseteq \mathcal{Y} ; \mathcal{L}$ is the space of 
TABLE I

NONLINEARITY MEASURES (NLM) COMPUTED FOR BOTH INPUT-OUTPUT PATHS

\begin{tabular}{|c|c|c|}
\hline Control Input & $\mathbf{N L M}_{1}$ & $\mathbf{N L M}_{2}$ \\
\hline Ethanol inlet flowrate & 0.2311 & 0.2079 \\
\hline Water inlet flowrate & 0.0479 & 0.0463 \\
\hline
\end{tabular}

continuous-time linear time-invariant systems; $\mathbf{x}_{L, 0}$ and $\mathbf{x}_{N, 0}$ are the initial values of the state of the linear operator $G$ and nonlinear operator $N$, respectively; and $\mathcal{S} \equiv\left\{\left(\mathbf{u}, \mathbf{x}_{N, 0}\right): \mathbf{u} \in\right.$ $\left.\mathcal{U}_{a}, \mathbf{x}_{N, 0} \in \mathcal{X}_{0, a}, N\left[\mathbf{u}, \mathbf{x}_{N, 0}\right] \in \mathcal{Y}_{a}\right\}$.

This nonlinearity measure is inspired by past definitions in the literature which are reviewed in a recent paper [13]. The above definition includes the optimization over the linear operator, which is included in many but not all past nonlinearity measures. Compared to the most complex nonlinearity measure that has been proposed [14], we drop an inner infinum over the initial state vector of the linear model as that optimization is not relevant in the determination of input-output models for use in control design. Removing that optimization also significantly reduces the computational complexity of the measure. We also replace the computationally expensive extensive sampling of all of the optimization variables proposed by some authors with a systematic grid of the input space by using staircase functions. These modifications produce a nonlinearity measure that is very inexpensive to compute even for singular distributed parameter systems consisting of multiple spatially distributed state variables, as in this study. As for past nonlinearity measures, a value of 0 indicates that the dynamical system is linear whereas a value of 1 indicates very high nonlinearity.

For the first nonlinearity measure, the outer infimum for $L$ is substituted by the model resulting of taking the coefficient mean of a set of 40 normalized step responses. Then, the inner supremum is computed between the models of this set, where $N$ is one of those models each time. The process is repeated for both inputs with respect to the only possible output. For the second nonlinearity measure, the inner supremum is computed in a completely analogous way, while the outer infimum is computed by taking $L$ to be one of the models of the set each time, and testing $L$ against all the other possible models ( $N$ in the inner loop).

The nonlinearity measures for the ethanol steam reformer, computed by one continuous simulation of the $1600 \times 1600$ DAE system, are reported in Table I. Both nonlinearity measures give similar values for each control input, with very low nonlinearity for the inlet water flowrate and mild nonlinearity for the inlet ethanol flowrate. These nonlinearities are sufficiently small that a linear control design would be suitable.

\section{LINEARITY Vs. NONLINEARITY DISCUSSION}

The nonlinearity measures indicated that the input-output dynamics for the ethanol steam reformer are well described by a linear time-invariant model. This section applies the numerical method in Section III to further explore nonlinearity in the system.
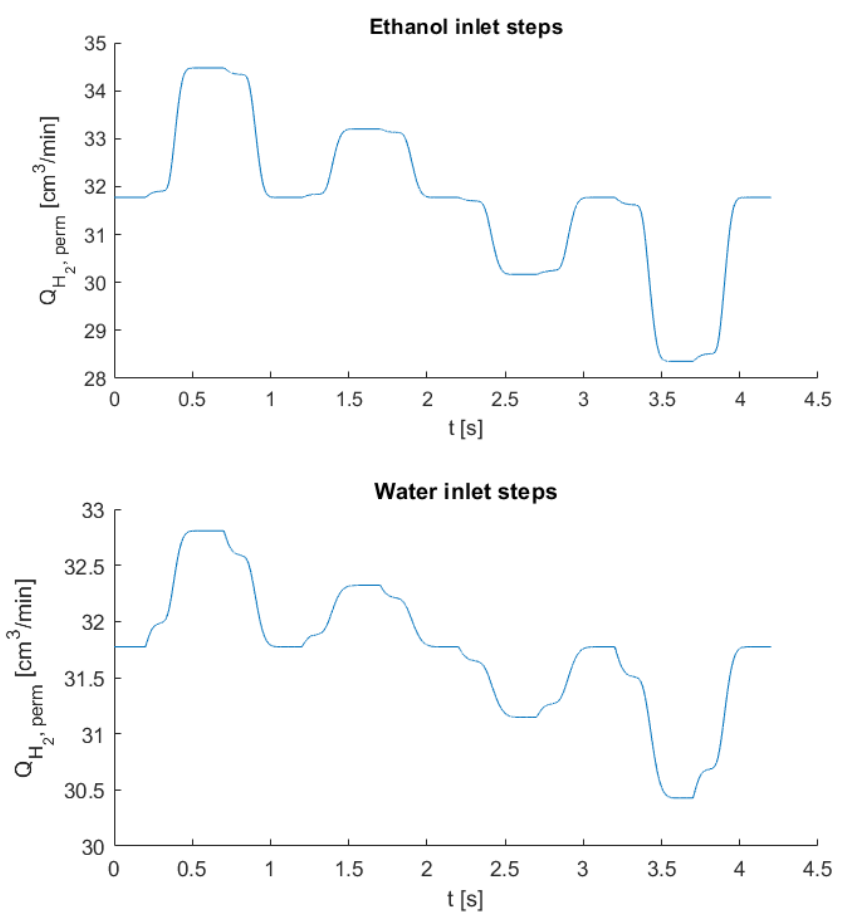

Fig. 2. The dynamic response of the system output (hydrogen permeate outflow) to consecutive steps in the ethanol (top) or water (bottom) inlet flowrates of $+100 \%,-100 \%,+50 \%,-50 \%,-50 \%,+50 \%,-100 \%$, $+100 \%$.

The dynamic response of the system output to steps in the control inputs show that the output has a small asymmetric nonlinear dependency on each control input (see Figs. 2 and 3 ). These simulation results for four step responses for each control input are consistent with the nonlinearity measures in Table I.

On the surface, these analyses indicating near linearity would seem inconsistent with the original model equations, which have significant nonlinearity in multiple key phenomena, including in the chemical reactions (3c)-(3f) and the membrane flow-pressure relationship (5a). The apparent discrepancy can be understood by inspection of the temporal evolution of the states at a different location in the system. Large nonlinearity is observed for some states in some locations. For example, the flowrates of $\mathrm{C}_{2} \mathrm{H}_{5} \mathrm{OH}$ and $\mathrm{CH}_{3} \mathrm{CHO}$ have a very large ${ }^{1}$ asymmetric nonlinear dependency on the control inputs at some spatial locations, such as observed in Fig. 3 ('State 1' and 'State 7'). The relationship between the control inputs on the spatially distributed state in the tubular reactor that is most relevant for determining the hydrogen permeate flowrate, however, has almost a perfectly linear response to the control inputs ('State 4' in Fig. 3). There is a square root nonlinearity between the hydrogen gas flowrate in the tube and the hydrogen permeate flowrate (5a), but the effect of that nonlinearity is small over the physically relevant range of control inputs. Hence the overall inputoutput relationship of the system is nearly linear.

${ }^{1}$ For example, a factor of three or more variation in the steady-state gain. 

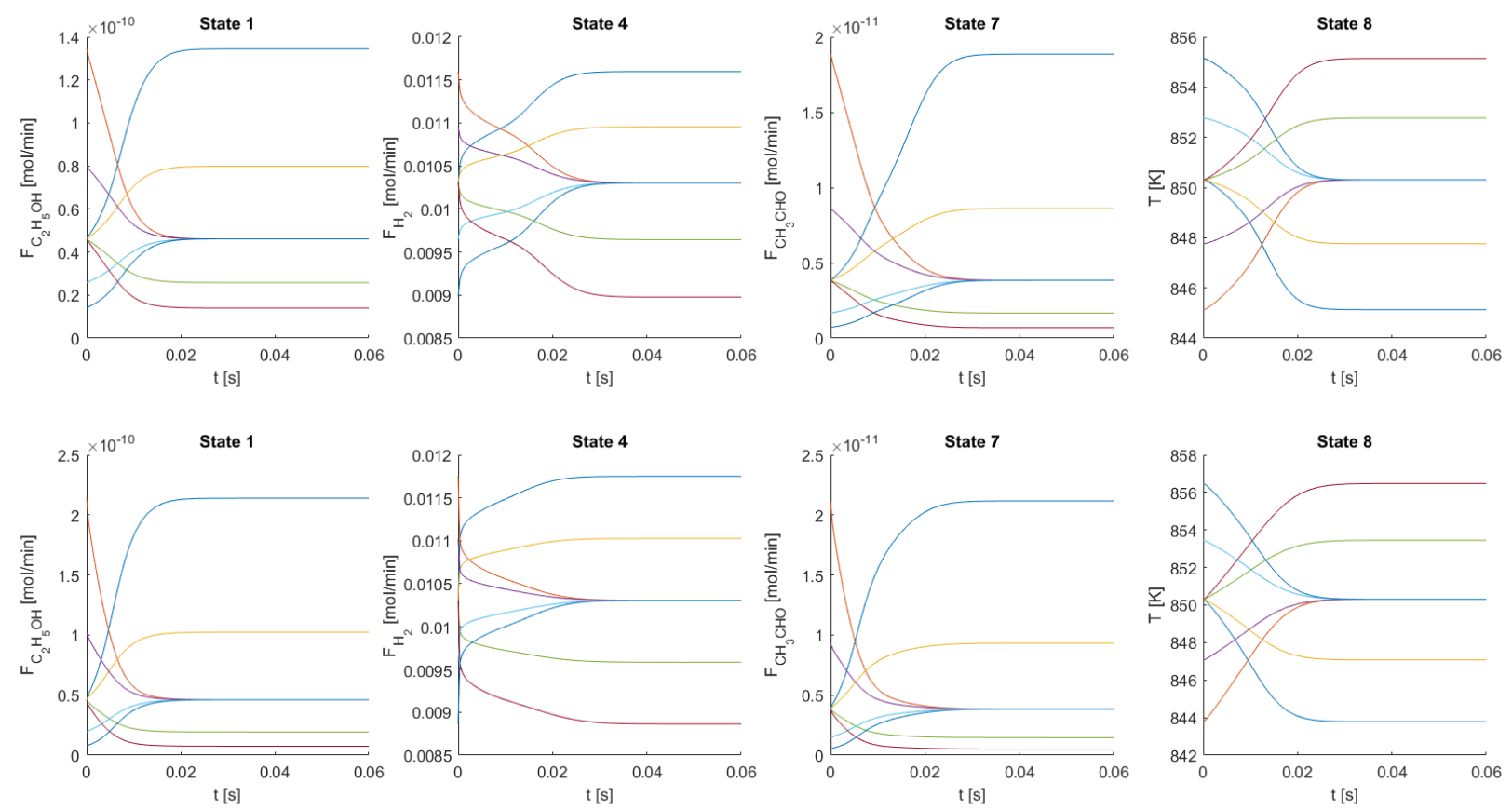

Fig. 3. The dynamic responses of some relevant states in the first stage at $z=l_{1} / 10$ (end of 1 st stage) to steps of $\pm 50 \%$ and $\pm 100 \%$ in the ethanol (top plots) or water (bottom plots) inlet flowrates.

\section{CONCLUSIONS}

This article analyzes the nonlinear dynamics for a physicsbased distributed parameter model for an ethanol steam reformer. Structural analysis is used to show that the model equations describe a singular distributed parameter system, and numerical simulation confirms that the states have many orders of magnitude variation in time scales. The analytical and numerical results fully resolve the extent of nonlinearity for ethanol steam reformers. While some states are a strongly nonlinear function of the control inputs, the output of interest - the hydrogen gas permeate flowrate - has only a weak asymmetric nonlinear dependency on the control inputs. Hence, a linear input-output process model is suitable for control design purposes. We construct a nominal linear inputoutput model that is shown to describe about $80 \%$ of the input-output behavior of the nonlinear singular distributed parameter system. The deviation in linearity of the inputoutput behavior of the ethanol stream reformer can be addressed by using a nonlinear uncertainty description and applying robust control design methods.

\section{REFERENCES}

[1] "Communication from the Commission to the European Parliament and the Council," European Commission, Brussels, Belgium, Tech. Rep., 2016.

[2] M. A. Abdalla, S. Hossain, O. B. Nisfindya, A. A. T., M. Dawoodb, and A. K. Azada, "Hydrogen production, storage, transportation and key challenges with applications: A review," Energy Conversion and Management, vol. 165, pp. 602-627, 2018

[3] M. David, C. Ocampo-Martinez, and R. Sanchez-Peña, "Advances in alkaline water electrolyzers: A review," Journal of Energy Storage, vol. 23, pp. 392-403, 2019.
[4] F. Zhang, P. Zhao, M. Niu, and J. Maddy, "The survey of key technologies in hydrogen energy storage," International Journal of Hydrogen Energy, vol. 41, pp. 14 535-14 552, 2016.

[5] V. M. García, E. López, M. Serra, J. Llorca, and J. Riera, "Dynamic modeling and controllability analysis of an ethanol reformer for fuel cell application," International Journal of Hydrogen Energy, vol. 35, pp. 9768-9775, 2010.

[6] V. M. Garcia, M. Serra, J. Llorca, and J. Riera, "Design of linear controllers applied to an ethanol steam reformer for PEM fuel cell applications," International Journal of Hydrogen Energy, vol. 38 , no. 18, pp. 7640-7646, 2013.

[7] D. Recio-Garrido, C. Ocampo-Martinez, and M. Serra-Prat, "Design of optimization-based controllers applied to an ethanol steam reformer for hydrogen production," International Journal of Hydrogen Energy, vol. 37, no. 15, pp. 11 141-11 156, 2012.

[8] E. Perez, M. Serra, C. Ocampo-Martinez, and J. Llorca, "Nonlinear model predictive control for hydrogen production in an ethanol steam reformer with membrane separation," in Proceedings of the IEEE Multi-Conference on Systems and Control, 2016, pp. 1155-1160.

[9] M. Serra, C. Ocampo-Martinez, M. Li, and J. Llorca, "Model predictive control for ethanol steam reformers with membrane separation," International Journal of Hydrogen Energy, vol. 42, no. 4, pp. 19491961, 2017

[10] M. Torchio, C. Ocampo-Martinez, L. Magni, M. Serra, R. D. Braatz, and D. M. Raimondo, "Fast model predictive control for hydrogen outflow regulation in ethanol steam reformers," in Proceedings of the American Control Conference, 2016, pp. 5044-5049.

[11] L. Dai, Singular Control Systems. Berlin-Heidelberg, Germany: Springer, 1989

[12] E. López, N. J. Divins, and J. Llorca, "Hydrogen production from ethanol over $\mathrm{Pd}-\mathrm{Rh} / \mathrm{CeO}_{2}$ with a metallic membrane reactor," Catalysis Today, vol. 193, no. 1, pp. 145-150, 2012.

[13] P. Reyero-Santiago, C. Ocampo-Martinez, R. Findeisen, and R. Braatz, "Nonlinearity measures for distributed parameter and descriptor systems," in Proceedings of the 21st IFAC World Congress, Berlin, Germany, July 12-17, 2020.

[14] A. Helbig, W. Marquardt, and F. Allgöwer, "Nonlinearity measures: Definition, computation and applications," Journal of Process Control, vol. 10, no. 2, pp. 113-123, 2000. 